\title{
Product of Several Two-Level Toeplitz Operators
}

\author{
Selcuk Koyuncu ${ }^{1} \&$ Kevin R. Bowman ${ }^{1}$ \\ ${ }^{1}$ Department of Mathematics, Morehouse College, Atlanta, Georgia, USA \\ Correspondence: Selcuk Koyuncu, Department of Mathematics, Morehouse College, Atlanta, Georgia, 830 West- \\ view Drive, USA. Tel: 1-404-909-1874. E-mail: skoyuncu@ morehouse.edu
}

Received: March 6, 2013 Accepted: April 6, 2013 Online Published: May 3, 2013

doi:10.5539/jmr.v5n2p61～URL: http://dx.doi.org/10.5539/jmr.v5n2p61

\begin{abstract}
In this paper we give a new proof to Theorem $1 \mathrm{in} \mathrm{Gu}$ and Zheng (1997). We then provide that $T_{f} T_{g}=0$ if and only if $f(z, w)$ or $g(z, w)$ is identically zero where $T_{f}$ and $T_{g}$ are two level Toeplitz operators with generating functions $f(z, w)$ and $g(z, w)$ being two variables bounded measurable functions on the unit circle. We also conjectured zero product of three two level Toeplitz operators.
\end{abstract}

Keywords: Toeplitz operator, bidisck, Hardy space

\section{Introduction}

Let $\mathbb{T}$ denote the boundary of the unit circle, with the standard Lebesgue measure, $L_{2}(\mathbb{T})$ be the Hilbert space of square-integrable functions. A bounded measurable function $f$ on $\mathbb{T}$ defines a multiplication operator $M_{f}$ on $L_{2}(\mathbb{T})$. Let $P$ be the projection from $L_{2}(\mathbb{T})$ onto the Hardy space $H_{2}(\mathbb{T})$. The Toeplitz operator with symbol $f$ is defined by $T_{f}=\left.P M_{f}\right|_{H_{2}}$, where "|" means restriction. A bounded operator on $H_{2}$ is Toeplitz if and only if its matrix representation, in the basis $\left\{z^{n}, n \geq 0\right\}$, has constant diagonals. These operators also have many natural relationships with other relevant mathematical objects such as sequence spaces, reproducing kernels, maximal ideals in Banach algebras, Wiener-Hopf processes, the commutant of the shift operator, etc. The algebra generated by Toeplitz operators is of particular importance and has generated a lot of interest in the recent years. It is easy to check that a Toeplitz operator is the zero operator if and only if its symbol is zero. Likewise, the product of two such operators is zero if and only if the symbol of one of them is zero (Brown, \& Halmos, 1963/1964).This result was generalized naturally for finitely many products of Toeplitz operators in (Aleman \& Vukotić, 2009). In this paper we provide a new proof to the existence result in (Gu \& Zheng, 1997). In (Gu \& Zheng, 1997), necessary and sufficient condition were given for when the product of two level Toeplitz operators is again Toeplitz. Our paper is organized as follows: In section 2, we discuss existence results provided in (Aleman \& Vukotić, 2009) for the product of two one level Toeplitz operators and zero product theorem. In section 3, we provide a new proof to product of several two-level Toeplitz operators and conditions for the zero product of two level Toeplitz operators. Finally, we state a conjecture concerning zero product of several two level Toeplitz operators.

\section{Product of One Level Toeplitz Matrices}

The set of all Toeplitz operators is certainly not commutative and certainly not closed under multiplication. A counterexample for both assertion is given by unilateral shift and its adjoint. Both $U$ and $U^{*}$ are Toeplitz operators, but the product $U^{*} U$ is not a Topelitz operators.

When is the product of two Toeplitz operator a Toeplitz operator? The answer is rarely. The proof depends on a simple but very useful equation involving matrix entries.

Lemma 1 Suppose $T_{f}$ and $T_{g}$ are Toeplitz operators where $f(z)$ and $g(z)$ are both bounded measureable functions on the unit circle. Write $C=T_{f} T_{g}$ and let $\left(c_{i j}\right)$ be the matrix of $C$. If the Fourier expansions of $f(z)$ and $g(z)$ are $f(z)=\sum_{k=-\infty}^{\infty} \hat{f}_{k}(z) z^{k}$ and $g(z)=\sum_{k=-\infty}^{\infty} \hat{g}_{k}(z) z^{k}$ where $\hat{f}(z)$ and $\hat{f}(z)$ are the Fourier coefficients of $f(z)$ and $g(z)$.

Then

$$
c_{i+1, j+1}=c_{i j}+\hat{f}_{i+1} \hat{g}_{-j-1},
$$

whenever $i, j \geq 0$. 
Proof. Since

$$
c_{i j}=\sum_{k=0}^{\infty} \hat{f}_{i-k} \hat{g}_{k-j},
$$

it follows that

$$
\begin{aligned}
c_{i+1, j+1} & =\sum_{k=0}^{\infty} \hat{f}_{i+1-k} \hat{g}_{k-j-1} \\
& =\hat{f}_{i+1} \hat{g}_{-j-1}+\sum_{k=1}^{\infty} \hat{f}_{i+1-k} \hat{g}_{k-j-1} \\
& =\hat{f}_{i+1} \hat{g}_{-j-1}+\sum_{k=0}^{\infty} \hat{f}_{i-k} \hat{g}_{k-j} \\
& =\hat{f}_{i+1} \hat{g}_{-j-1}+c_{i j} .
\end{aligned}
$$

Theorem 2 A necessary and sufficient condition that the product that the product $T_{f} T_{g}$ of two Toeplitz operators be a Toeplitz operators is that either $f(z)$ be co-analytic or $g(z)$ be analytic; if the condition is satisfied, then $T_{f} T_{g}=T_{f g}$.

Proof. If $g(z)$ is analytic, then

$$
T_{f} T_{g} h=T_{f}(g h)=P(f g h)=T_{f g} h
$$

for all $h(z) \in H_{2}$, so that $T_{f} T_{g}=T_{f g}$. If $f(z)$ is co-analytic, then $\bar{f}(z)$ is analytic, and therefore

$$
T_{f} T_{g}=\left(T_{\bar{g}} T_{\bar{f}}\right)^{*}=T_{\bar{f} \bar{g}}^{*}=T_{f g} .
$$

Theorem 3 (Riesz, F. \& Riesz, M., 1916) Let $F$ be analytic on $\mathbb{D}$ and $L^{1}$-bounded, i.e, $F \in H^{1}(\mathbb{D})$. Assume that $F \neq 0$ and set $f=\lim _{r \rightarrow 1^{-}} F_{r}$. Then $\log |f| \in L^{1}(\mathbb{T})$. In particular, $f$ does not vanish on a set of positive measure.

Theorem 4 Let $f(z)$ and $g(z)$ be bounded measurable functions on the unit disk. Then $T_{f} T_{g}=0$ if and only if Either $f(z)=0$ or $g(z)=0$.

Proof. Sufficiency if trivial. If, conversely, $T_{f} T_{g}=0$, then, since 0 is a Toeplitz operator, it follows from Theorem 2 that either $f(z)$ is co-analytic or $g(z)$ is analytic and that $f(z) g(z)=0$. By Theorem 3 , since a non-zero analytic function, cannot vanish on a set of positive measure, it follows that if $f(z)$ is co-analytic, then $g(z)=0$, and if $g(z)$ is analytic, then $f(z)=0$.

Remark 1 The following Theorem provides a new proof to Theorem 1 in (Gu \& Zheng, 1997). Our proof is not based on operator theoretical techniques. We use one variable approach which was used in (Brown \& Halmos, 1963/1964). Therefore, we believe our proof is simple to understand.

\section{Product of Two Level Toeplitz Operators}

Theorem 5 Let $f(z, w)=\sum_{(k, l) \in \mathbb{Z}^{2}} \hat{f}_{k, l} z^{k} w^{l} \in L_{\infty}\left(\mathbb{T}^{2}\right)$ and $g(z, w)=\sum_{(k, l) \in \mathbb{Z}^{2}} \hat{g}_{k, l} z^{k} w^{l} \in L_{\infty}\left(\mathbb{T}^{2}\right)$ where $\hat{f}_{k, l}$ and $\hat{g}_{k, l}$ are the Fourier coefficients of $f(z, w)$ and $g(z, w)$, respectively.

$$
T_{f} T_{g}=T_{f g}
$$

if and only if one of the followings holds.

(i) $f(z, w)$ is co-analytic in $z$ and $f(z, w)$ is co-analytic in $w$;

(ii) $f(z, w)$ is co-analytic in $z$ and $g(z, w)$ is analytic in $w$;

(iii) $g(z, w)$ is analytic in $z$ and $f(z, w)$ is co-analytic in $w$;

(iv) $g(z, w)$ is analytic in $z$ and $g(z, w)$ is analytic in $w$.

Proof. Suppose that $T_{f} T_{g}=T_{f g}$. Our first strategy is to show that $f(z, w)$ is co-analytic in $z$ or $g(z, w)$ is analytic in $z$. Next, we will show $f(z, w)$ is co-analytic in $w$ or $g(z, w)$ is analytic in $w$. Ordering $\mathbb{N}_{0}^{2}$ lexicographically, i.e,

$$
\{(0,0),(0,1),(0,2) \ldots,(1,0),(1,1),(1,2), \ldots,(i, 0),(i, 1),(i, 2), \ldots\} .
$$


Then write $T_{f}=\left(F_{i-j}\right)_{i, j=0}^{\infty}$ and $T_{g}=\left(G_{i-j}\right)_{i, j=0}^{\infty}$ where

$$
\begin{aligned}
F_{i} & =\left[\begin{array}{cccc}
\hat{f}_{i, 0} & \hat{f}_{i,-1} & \hat{f}_{i,-2} & \cdots \\
\hat{f}_{i, 1} & \hat{f}_{i, 0} & \hat{f}_{i,-1} & \cdots \\
\hat{f}_{i, 2} & \hat{f}_{i, 1} & \ddots & \ddots \\
\vdots & \ddots & \ddots & \ddots
\end{array}\right], F_{-i}=\left[\begin{array}{cccc}
\hat{f}_{-i, 0} & \hat{f}_{-i,-1} & \hat{f}_{-i,-2} & \cdots \\
\hat{f}_{-i, 1} & \hat{f}_{-i, 0} & \hat{f}_{-i,-1} & \cdots \\
\hat{f}_{-i, 2} & \hat{f}_{-i, 1} & \ddots & \ddots \\
\vdots & \ddots & \ddots & \ddots
\end{array}\right] \\
G_{i} & =\left[\begin{array}{cccc}
\hat{g}_{i, 0} & \hat{g}_{i,-1} & \hat{g}_{i,-2} & \cdots \\
\hat{g}_{i, 1} & \hat{g}_{i, 0} & \hat{g}_{i,-1} & \cdots \\
\hat{g}_{i, 2} & \hat{g}_{i, 1} & \ddots & \ddots \\
\vdots & \ddots & \ddots & \ddots
\end{array}\right], G_{-i}=\left[\begin{array}{cccc}
\hat{g}_{-i, 0} & \hat{g}_{-i,-1} & \hat{g}_{-i,-2} & \cdots \\
\hat{g}_{-i, 1} & \hat{g}_{-i, 0} & \hat{g}_{-i,-1} & \cdots \\
\hat{g}_{-i, 2} & \hat{g}_{-i, 1} & \ddots & \ddots \\
\vdots & \ddots & \ddots & \ddots
\end{array}\right] .
\end{aligned}
$$

(1) yields that, $F_{i+1} G_{-j-1}=0$ for all $i, j \in \mathbb{N}_{0}$. Thus $F_{i+1}=0$ for all $i \in \mathbb{N}_{0}$ or $G_{-j-1}=0$ for all $j \in \mathbb{N}_{0}$ by Theorem 4. If $F_{i+1}=0$ then $\hat{f}_{i+1, k}=0$ for all $(i, k) \in \mathbb{N}_{0} \times \mathbb{Z}$, i.e, $f(z, w)$ is co-analytic in $z$. If $G_{-j-1}=0$ then $\hat{g}_{-j-1, k}=0$ for all $(j, k) \in \mathbb{N}_{0} \times \mathbb{Z}$, i.e, $g(z, w)$ is analytic in $z$. Thus we can conclude that (1) gives us $f(z, w)$ is co-analytic in $z$ or $g(z, w)$ is analytic in $z$. In order to see $f(z, w)$ is co-analytic in $w$ or $g(z, w)$ is analytic in $w$, it suffices to use reverse lexicographical ordering on $\mathbb{N}_{0}^{2}$. Finally we repeat the same argument to get the desired claim. We now have that $f(z, w)$ is co-analytic in $w$ or $g(z, w)$ is analytic in $w$. Combining $f(z, w)$ is co-analytic in $z$ or $g(z, w)$ is analytic in $z$ and $f(z, w)$ is co-analytic in $w$ or $g(z, w)$ is analytic in $w$, we get four different cases given in Theorem 5 .

Conversely, if ( $i$ ) holds, i.e, $f(z, w)$ is co-analytic in $z$ and $f(z, w)$ is co-analytic in $w$ then we will prove that $T_{f} T_{g}$ is Toeplitz. Let $s, t, r \in \mathbb{N}_{0}^{2}$. Write

$$
\left(T_{f} T_{g}\right)_{(s, t)}=\sum_{k \in \mathbb{N}_{0}^{2}} \hat{f}_{s-k} \hat{g}_{k-t}
$$

Since $k_{1}-s_{1} \geq 0$ and $k_{2}-s_{2} \geq 0$, then (2) becomes

$$
\left(T_{f} T_{g}\right)_{(s, t)}=\sum_{k \in\left(\left(s_{1}, 0\right)+\mathbb{N}_{0}^{2} \cap\left(s_{2}, 0\right)+\mathbb{N}_{0}^{2}\right)} \hat{f}_{s-k} \hat{g}_{k-t}
$$

Now write

$$
\left(T_{f} T_{g}\right)_{(s+r, t+r)}=\sum_{k \in\left(\left(s_{1}+s_{1}, 0\right)+\mathbb{N}_{0}^{2} \cap\left(s_{2}+r_{2}, 0\right)+\mathbb{N}_{0}^{2}\right)} \hat{f}_{s-k+r} \hat{g}_{k-t-r}
$$

Letting $\tilde{k}=k-r$, we get $s_{1} \leq \tilde{k}_{1}$ and $s_{2} \leq \tilde{k}_{2}$. Thus (4) becomes

$$
\left(T_{f} T_{g}\right)_{(s+r, t+r)}=\sum_{\tilde{k} \in\left(s_{1}, s_{2}\right)+\mathbb{N}_{0}^{2}} \hat{f}_{s-\tilde{k}} \hat{g}_{\tilde{k}-t}
$$

Thus this proves that (3) and (5) are equal.

Now suppose that (ii) holds, i.e, $f(z, w)$ is co-analytic in $z$ and $g(z, w)$ is analytic in $w$. Write

$$
\left(T_{f} T_{g}\right)_{(s, t)}=\sum_{k \in \mathbb{N}_{0}^{2}} \hat{f}_{s-k} \hat{g}_{k-t}
$$

Since $k_{1}-s_{1} \geq 0$ and $k_{2}-t_{2} \leq 0$, (6) becomes

$$
\left(T_{f} T_{g}\right)_{(s, t)}=\sum_{k \in \Theta=\left(\left(s_{1}, 0\right)+\mathbb{N}_{0}^{2} \cap\left(0, t_{2}\right)+\mathbb{N}_{0}^{2}\right)} \hat{f}_{s-k} \hat{g}_{k-t}
$$

Now write

$$
\left(T_{f} T_{g}\right)_{(s+r, t+r)}=\sum_{k \in\left(\left(s_{1}+s_{1}, 0\right)+\mathbb{N}_{0}^{2} \cap\left(0, t_{2}+r_{2}\right)+\mathbb{N}_{0}^{2}\right)} \hat{f}_{s-k+r} \hat{g}_{k-t-r}
$$

Letting $\tilde{k}=k-r$, we get $s_{1} \leq \tilde{k}_{1}, \tilde{k}_{2} \leq t_{2}, \tilde{k}_{2} \leq-r_{2}$ and $-r_{1} \leq \tilde{k}_{1}$. This implies that $\tilde{k} \in \Lambda=\left(\left(s_{1},-r_{2}\right)+\mathbb{N}_{0}^{2} \cap\right.$ $\left.\left(-r_{2}, t_{2}\right)+\mathbb{N}_{0}^{2}\right) \cap\left(\left(s_{1}, 0\right)+\mathbb{N}_{0}^{2} \cap\left(0, t_{2}\right)+\mathbb{N}_{0}^{2}\right)$. Thus (8) becomes

$$
\left(T_{f} T_{g}\right)_{(s+r, t+r)}=\sum_{\tilde{k} \in \Lambda} \hat{f}_{s-\tilde{k}} \hat{g}_{\tilde{k}-t} .
$$


Note that since $\Theta=\Lambda$, then (7) and (9) are equal.

Theorem 5 Let $f(z, w)$ and $g(z, w)$ be in $L_{\infty}\left(\mathbb{T}^{2}\right)$. Then $T_{f} T_{g}=0$ if and only if either $f(z, w)=0$ or $g(z, w)=0$.

Proof. Sufficiency is trivial. Conversely, if $T_{f} T_{g}=0$, then, since 0 is a Toeplitz operator, it follows from Theorem 5 that one of the four cases hold and that $f(z, w) g(z, w)=0$. In the case where $f(z, w)$ is co-analytic in $z$ and $w$ or $g(z, w)$ is analytic in $z$ and $w$, then Theorem 3 applies that Since a non-zero analytic function, cannot vanish on a set of positive measure, it follows that if $f(z, w)$ is co-analytic in $z$ and $w$, then $g(z, w)=0$, and if $g(z, w)$ is analytic in $z$ and $w$, then $f(z, w)=0$. In the case where $f(z, w)$ is co-analytic in $z$ and $g(z, w)$ is analytic in $w$, we have that $f(z, w) g(z, w)=0$ a.e on $\mathbb{T}^{2}$. Let $E \times F \subseteq \mathbb{T} \times \mathbb{T}$ be the zero set of $f$ and $E$ have positive measure in $\mathbb{T}$. Since $f(z, w)$ is co-analytic in $z$ and $f(z, w) \in L_{\infty}\left(\mathbb{T}^{2}\right)$, then for each fixed $w \in \mathbb{T}$, we have that $f(z, w) \in H^{2}(\mathbb{T})$. Thus $f(z, w)=0$ a.e for $z \in \mathbb{T}$ and $w \in F$, i.e, $E=F$ and hence $f(z, w)=0$ on $\mathbb{T} \times F$. If the set $F$ has full measure in $\mathbb{T}$, then $f(z, w)=0$ on $\mathbb{T}^{2}$. However, assume that $F$ is not of full measure in $\mathbb{T}$, then $g(z, w)=0$ on $\mathbb{T}^{2}-\mathbb{T} \times F=\mathbb{T} \times(\mathbb{T}-F)$ and $\mathbb{T}-F$ has positive measure. But $g(z, w)$ is analytic in $w$ and for fixed $z \in \mathbb{T}$, we have $g(z, w) \in H^{2}(\mathbb{T})$. Notice that this implies $g(z, w)=0$ a.e for $w \in \mathbb{T}$. Thus $\mathbb{T}-F=\mathbb{T}$, i.e, $g(z, w)=0$ on $\mathbb{T}^{2}$.

We now state the following conjecture.

Conjecture Let $f_{1}\left(z_{1}, z_{2}\right), f_{2}\left(z_{1}, z_{2}\right)$ and $f_{3}\left(z_{1}, z_{2}\right)$ be in $L_{\infty}\left(\mathbb{T}^{2}\right)$. Then $T_{f_{1}} T_{f_{2}} T_{f_{3}}=0$ if and only if at least one of $f_{i}=0$ for $1 \leq i \leq 3$.

\section{Acknowledgements}

We would like to thank to the referees for their helpful suggestions.

\section{References}

Aleman, A., \& Vukotić, D. (2009). Zero products of Toeplitz operators. Duke Math. J., 148(3), $373-403$. http://dx.doi.org/10.1215/00127094-2009-029

Brown, A., \& Halmos, P. R. (1963/1964). Algebraic properties of Toeplitz operators. J. Reine Angew. Math., 213 , 89-102.

Gu, C., \& Zheng, D. (1997). The semi-commutator of Toeplitz operators on the bidisc. J. Operator Theory, 38(1), 173-193.

Riesz, F., \& Riesz, M. (1916). Uber die randwerte einer analytischen funktion. Quatrieme Congres des Mathematiciens Scandinaves, Stockholm, pp. 27-44. 